\title{
Genome Resource for the Huanglongbing Causal Agent 'Candidatus Liberibacter asiaticus' Strain AHCA17 from Citrus Root Tissue in California, USA
}

\author{
Weili Cai, ${ }^{1,2}$ Schyler Nunziata, ${ }^{1}$ Stefano Costanzo, ${ }^{1}$ Lucita Kumagai, ${ }^{3}$ John Rascoe, ${ }^{1}$ \\ and Michael J. Stulberg ${ }^{1, t}$ \\ ${ }^{1}$ United States Department of Agriculture-Animal and Plant Health Inspection Service, Plant Protection \\ and Quarantine, Science and Technology, Beltsville, MD \\ ${ }^{2}$ Department of Entomology and Plant Pathology, North Carolina State University, Raleigh, NC \\ ${ }^{3}$ Plant Pests Diagnostics Branch, California Department of Food and Agriculture, Sacramento, CA
}

\begin{abstract}
'Candidatus Liberibacter asiaticus' is the unculturable causative agent of citrus huanglongbing disease. Here, we report the first citrus root metagenome sequence containing the draft genome of ' $\mathrm{Ca}$. L. asiaticus' strain AHCA17, obtained from a pummelo tree in California. The assembled genome was $1.2 \mathrm{Mbp}$ and resulted in 37 contigs $\left(\mathrm{N}_{50}=158.7 \mathrm{kbp}\right)$ containing 1,057 predicted open reading frames and 45 RNA-coding genes. This draft genome will provide a valuable resource in further study of 'Ca. L. asiaticus' genome diversity and pathogen epidemiology.
\end{abstract}

'Candidatus Liberibacter asiaticus' is an unculturable bacteria and the causal agent of a serious citrus disease, huanglongbing (HLB) (Bové 2006; Jagoueix et al. 1994). HLB causes great economic losses annually in citrus production worldwide (Gottwald et al. 2007). Detection of ' $C a$. L. asiaticus' is difficult due to uneven distribution in citrus tissues, low pathogen titer, and latency between infections and symptoms. A previous study showed that roots were one of the first tissues to show disease symptoms (Johnson et al. 2014). Currently, publicly available ' $\mathrm{Ca}$. L. asiaticus' genomes from citrus samples were all obtained from foliar tissue (Cai et al. 2018; Dai et al. 2019; Wu et al. 2015; Zheng et al. 2014, 2018). Sequencing a 'Ca. L. asiaticus' genome from root tissue presents new challenges, considering the complex microbiota associated with this tissue (Zhang et al. 2017). Making additional ' $\mathrm{Ca}$. L. asiaticus' genomes public provides a valuable supporting resource to understanding ' $\mathrm{Ca}$. L. asiaticus' genome diversity, pathogen movement, and host interaction. Thus, here, we report the first draft whole-genome sequence of ' $\mathrm{Ca}$. L. asiaticus' strain AHCA17, obtained directly from the root tissue of a pummelo tree (Citrus maxima) in Orange County, CA.

Genomic DNA of strain AHCA17 was extracted from root tissue using the DNeasy PowerPlant Pro kit (Qiagen, Valencia, CA, U.S.A.). The pathogen titer level was estimated using HLB nrdB-based real-time quantitative PCR, producing a threshold cycle value of 14.36 (Zheng et al. 2016), which signifies relatively high pathogen levels that are critical for successful sequencing. The $2 \times 300$-bp Illumina paired-end sequencing was performed on an Illumina MiSeq platform (Illumina, Inc., San Diego, CA, U.S.A.). The paired-end sequencing libraries were prepared using an Illumina Nextera DNA Flex library prep kit.

${ }^{\dagger}$ Corresponding author: M. J. Stulberg; michael.stulberg@usda.gov

The findings and conclusions in this publication are those of the authors and should not be construed to represent any official USDA or U.S. Government determination or policy.

The author(s) declare no conflict of interest.

Accepted for publication 19 September 2019.

This article is in the public domain and not copyrightable. It may be freely reprinted with customary crediting of the source. The American Phytopathological Society, 2020.
Funding

This research was supported by the United States Department of Agriculture (USDA) Animal and Plant Health Inspection Service.

Keywords

citrus, metagenomics, prokaryotes 
Table 1. Comparison of bacterial diversity between a leaf sample and this root sample

\begin{tabular}{|c|c|c|c|c|}
\hline \multirow[b]{2}{*}{ Taxon $^{c}$} & \multicolumn{2}{|c|}{ Raw reads ${ }^{a}$} & \multicolumn{2}{|c|}{$\begin{array}{c}\text { Percentage of } \\
\text { previous taxon }(\%)^{b}\end{array}$} \\
\hline & Leaf & Root & Leaf & Root \\
\hline Bacteria & $1,081,337$ & $1,644,680$ & 4.19 & 10.28 \\
\hline Proteobacteria & 448,570 & 705,316 & 41.48 & 42.88 \\
\hline$\alpha$-Proteobacteria & 58,495 & 363,619 & 13.04 & 51.55 \\
\hline Rhizobiales & 36,633 & 274,884 & 62.63 & 75.60 \\
\hline Rhizobiaceae & 23,783 & 95,018 & 64.92 & 34.57 \\
\hline Liberibacter & 21,805 & 51,792 & 91.68 & 54.51 \\
\hline
\end{tabular}

a Total leaf sample (Cai et al. 2018) raw MiSeq reads numbered 25,830,723 and 15,992,407 for the root sample. Mapped using Kraken v1.1.1.

b Percentage of reads composing immediately higher taxon (Bacteria indicates percentage of total reads).

c Taxon lineage from Bacteria (superkingdom) to Liberibacter (genus).

In total, $3.19 \times 10^{7}$ reads with a mean of $269 \mathrm{bp} / \mathrm{read}$ were generated from MiSeq sequencing. Kraken (version 1.1.1) (Wood and Salzberg 2014) analysis revealed that $10.28 \%$ of the reads mapped to bacteria and not plant host (Table 1; mapped to complete bacterial, archeal, and viral Refseq genomes as of 18 July 2019). This is in contrast to a citrus leaf midrib sample we sequenced $\left(5.16 \times 10^{7}\right.$ reads with the same mean read length), which only had $4.19 \%$ of the reads map to bacteria (Cai et al. 2018). Interestingly, within bacteria, the Proteobacteria phylum was represented similarly between the root and leaf sample (41.48 and $42.88 \%$, respectively). Within proteobacteria, there is an increase in diversity in the root sample compared with the leaf, exemplified by Liberibacter spp. representing $54.51 \%$ of the Rhizobiaceae family in the root sample and representing $91.68 \%$ in the leaf. The larger diversity of closely related organisms in the root led us to a map-to-pathogen, then assemble approach to analyzing the data.

Using the 'Ca. L. asiaticus' genome strain psy62 as a reference, 104,637 reads with $25,536,511$ bases were aligned using Bowtie2 (version 2.3.0) covering $99.8 \%$ of the psy62 genome (Duan et al. 2009; Langmead and Salzberg 2012). Aligned reads were assembled using the SPAdes assembler (version 3.10.0) (Nurk et al. 2013) generating 37 contigs $\geq 500 \mathrm{bp}$, ranging from 500 to $266,672 \mathrm{bp}\left(\mathrm{N}_{50}=158.7 \mathrm{kbp}\right.$ ), with 24.56 average coverage. The contigs comprised a total length of $1,208,622 \mathrm{bp}$, with a $\mathrm{G}+\mathrm{C}$ content of $36.39 \%$, and aligned with $98.08 \%$ of the complete psy62 genome. Annotation was performed by prokka (version 1.13.3) (Seemann 2014). The genome included 1,057 predicted open reading frames and 45 RNA-coding genes.

This Whole-Genome Shotgun project has been deposited at DNA Data Bank of Japan/ European Nucleotide Archive/GenBank under the accession VNFL00000000. The version described in this article is version VNFL01000000. Data has also been deposited in the Sequence Read Archive database (PRJNA552562).

\section{Literature Cited}

Bové, J. M. 2006. Huanglongbing: A destructive, newly emerging, century-old disease of citrus. J. Plant Pathol. 88:7-37.

Cai, W., Yan, Z., Rascoe, J., and Stulberg, M. J. 2018. Draft whole-genome sequence of "Candidatus Liberibacter asiaticus" strain TX1712 from citrus in Texas. Genome Announce. 6:e00554-18.

Dai, Z., Wu, F., Zheng, Z., Yokomi, R., Kumagai, L., Cai, W., Rascoe, J., Polek, M., Chen, J., and Deng, X. 2019. Prophage diversity of 'Candidatus Liberibacter asiaticus' strains in California. Phytopathology 109:551-559.

Duan, Y., Zhou, L., Hall, D. G., Li, W., Doddapaneni, H., Lin, H., Liu, L., Vahling, C. M., Gabriel, D. W., Williams, K. P., Dickerman, A., Sun, Y., and Gottwald, T. 2009. Complete genome sequence of citrus huanglongbing bacterium, 'Candidatus Liberibacter asiaticus' obtained through metagenomics. Mol. Plant-Microbe Interact. 22:1011-1020.

Gottwald, T. R., da Graça, J. V., and Bassanezi, R. B. 2007. Citrus huanglongbing: The pathogen and its impact. Plant Health Prog. 8.

Jagoueix, S., Bové, J. M., and Garnier, M. 1994. The phloem-limited bacterium of greening disease of citrus is a member of the $\alpha$ subdivision of the Proteobacteria. Int. J. Syst. Bacteriol. 44:379-386.
Johnson, E. G., Wu, J., Bright, D. B., and Graham, J. H. 2014. Association of 'Candidatus Liberibacter asiaticus' root infection, but not phloem plugging with root loss on huanglongbing-affected trees prior to appearance of foliar symptoms. Plant Pathol. 63:290-298.

Langmead, B., and Salzberg, S. L. 2012. Fast gapped-read alignment with Bowtie 2. Nat. Methods 9:357-359.

Nurk, S., Bankevich, A., Antipov, D., Gurevich, A., Korobeynikov, A., Lapidus, A., Prjibelsky, A., Pyshkin, A., Sirotkin, A., Sirotkin, Y., Stepanauskas, R., McLean, J., Lasken, R., Clingenpeel, S. R., Woyke, T., Tesler, G., Alekseyev, M. A., and Pevzner, P. A. 2013. Assembling genomes and mini-metagenomes from highly chimeric reads. Pages 158-170 in: Research in Computational Molecular Biology. M. Deng, R. Jiang, F. Sun, and X. Zhang, eds. RECOMB 2013. Lecture Notes in Computer Science, vol 7821. Springer, Berlin, Heidelberg, Germany.

Seemann, T. 2014. Prokka: Rapid prokaryotic genome annotation. Bioinformatics 30: 2068-2069.

Wood, D. E., and Salzberg, S. L. 2014. Kraken: Ultrafast metagenomic sequencing classification using exact alignments. Genome Biol. 15:R46.

Wu, F., Zheng, Z., Deng, X., Cen, Y., Liang, G., and Chen, J. 2015. Draft genome sequence of "Candidatus Liberibacter asiaticus" from Diaphorina citri in Guangdong, China. Genome Announce. 3:e01316-15. 
Zhang, Y., Xu, J., Riera, N., Jin, T., Li, J., and Wang, N. 2017. Huanglongbing impairs the rhizosphere-to-rhizoplane enrichment process of the citrus root-associated microbiome. Microbiome 5:97.

Zheng, Z., Bao, M., Wu, F., Van Horn, C., Chen, J., and Deng, X. 2018. A type 3 prophage of 'Candidatus Liberibacter asiaticus' carrying a restriction-modification system. Phytopathology 108:454-461.
Zheng, Z., Deng, X., and Chen, J. 2014. Draft genome sequence of "Candidatus Liberibacter asiaticus" from California. Genome Announce. 2: e00999-14.

Zheng, Z., Xu, M., Bao, M., Wu, F., Chen, J., and Deng, X. 2016. Unusual five copies and dual forms of $n r d B$ in "Candidatus Liberibacter asiaticus": Biological implications and PCR detection application. Sci. Rep. 6:39020. 